\title{
New Perspectives in Human Movement Variability
}

Movement variability is defined as the normal variations that occur in motor performance across multiple repetitions of a task. ${ }^{2}$ Bernstein $^{1}$ described movement variability quite eloquently as "repetition without repetition." Traditionally, movement variability has been linked to noise and error, being considered to be random and independent. This theoretical approach blends well with traditional statistical and assessment methods of movement variability that assume randomness and independence of observations. However, numerous studies have indicated that when movement is observed over time variations are closely related with each other neither being random nor independent. Practically, traditional methods can mask the temporal structure of movement variability and contain little information about how movement changes over time.

Importantly, a growing body of literature has shown that the variations seen in a wide variety of physiological systems may offer insight into the control of these motor systems. For example, they can enhance our understanding of the effect of various pathologies on movement allowing us to develop better prognostic and diagnostic biomedical engineering tools. Thus, many new methods have been presented recently to assess the temporal structure of movement variability. These nonlinear methods can also allow us to develop alternative theoretical models to guide experimental designs and scientific inquiry.

Therefore, in this special issue we provide a thorough view of the state-of-the-art methods currently used in the mainstream biomechanics for evaluating and quantifying human movement variability. These new perspectives in human movement variability are further elaborated in the light of the temporal structure of movement variability. It is our hope that this special issue will provide a thorough background in understanding of the fundamental concepts of human movement variability and their utility in solving particular problems in motor control, and related peripheral topics and fields in the biomedical engineering. Our collection of 14 papers from leading laboratories highlights these advances in distinct human movement arenas.

The articles featured in this special issue address several different motor control systems utilizing various nonlinear dynamical systems tools. The first article discusses the theory of optimal movement variability (Kaipust, McGrath, Mukherjee, and Stergiou) investi- gating the complexity and predictability of time series data of gait characteristics by assessing fractal indexes in a dual task paradigm (detrended fluctuation analysis). Similarly, the second and third articles explore the fluctuation dynamics of cycling (Warlop, Bollens, Crevecoeur, Detrembleur, and Lejeune) and eye saccades using a regime-switching approach (Wong and Shelhamer). Fractal fluctuations of center-of-pressure data were determined in the fourth paper to better understand the cascade dynamics (Palatinus, Dixon, and KeltyStephen). The fifth (Ihlen and Vereijken) and sixth (Kuznetsov, Bonnette, Gao, and Riley) articles propel us into the new frontier of the multifractal scaling analyses per-

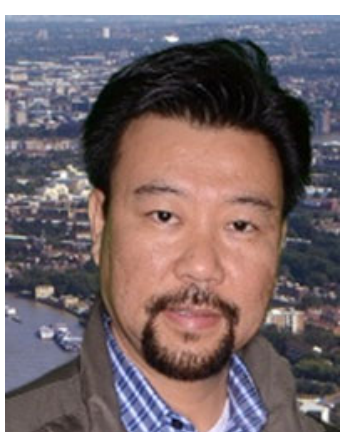

Thurmon Lockhart

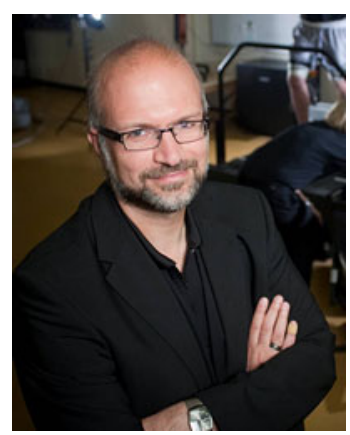

Nick Stergiou haps more fitting for postural stability modeling than the monofractal scaling methods. The seventh article investigates the utility of fractal scaling analysis in biofeedback training during walking (Tirosh, Cambell, Begg, and Sparrow). The next three articles employed varied linear and nonlinear methods (maximal Lyapunov exponents using Wolf's and Rosenstein's methods, approximate entropy, and detrended fluctuation analysis) to explore the notions of complexity and stability. The eighth article (Huisinga, Mancini, George, and Horak) probed the movement variability of walking using a trunk accelerometer data of patients with Multiple Sclerosis and healthy controls. The ninth article (Nessler, McMillan, Schoulten, Shallow, Stewart, and De Leone) elaborates on the nonlinear dynamics of desynchronized gait with an interesting walking protocol - side-by-side treadmill walking. The tenth article (Myers, Johanning, Pipinos, Schmid, and Stergiou) evaluates gait variability changes post vascular occlusion using complementary linear and nonlinear analyses. The next set of articles describes independent linear and nonlinear methods to assess characteristics 
related to movement variability. The eleventh article (Kurz, Arpin, Davies, and Harbourne) elaborates on the stochastic and deterministic components within the COP movement variability of children with balance impairments using a Langevin equation methodology. The next article (Ramdani, Tallon, Bernard, and Blain) discusses an interesting approach of differentiating older fallers and non-fallers utilizing the recurrence quantification analysis. The thirteenth article (Roerdink, Ridderikhoff, Peper, and Beek) exploits informational timing and neuromuscular properties in the control of rhythmic movements. In the last article of our special issue, footfall placement variability is investigated using a novel Fourier based variability concept (Socie, Sandroff, Pula, Hsiao-Wecksler, Motl, and Sosnoff).

We hope that you will find these articles stimulating and informative, and timely in your research in understanding human movement variability.

\section{ACKNOWLEDGMENTS}

We acknowledge grant support from the NSFInformation and Intelligent Systems (IIS) and Smart Health and Wellbeing-1065442 and 1065262 (Lockhart), and NIH R01AG034995 and NASA EPSCOR (Stergiou).

\section{REFERENCES}

${ }^{1}$ Bernstein, N. A. On dexterity and its development. In: Dexterity and its Development, edited by M. L. Latash, and M. T. Turvey. Mahwah: Lawrence Erlbaum Associates, 1996, pp. 1-244.

${ }^{2}$ Stergiou, N., and L. M. Decker. Human movement variability, nonlinear dynamics, and pathology: is there a connection? Hum. Mov. Sci. 30(5):869-888, 2011.

\section{THURMON LOCKHART}

Department of Industrial and Systems Engineering, Virginia Tech,

Blacksburg, VA, USA

School of Biomedical Engineering and Sciences, Virginia Tech/Wake Forest University, Virginia Tech, Blacksburg, VA, USA

Electronic mail: lockhart@vt.edu

Nick Stergiou

Nebraska Biomechanics Core Facility and the Center for Research in Biomechanics, College of Education and College of Public Health University of Nebraska at Omaha and University of Nebraska Medical Center,

Omaha, NE, USA 